\title{
Design of an adaptive state feedback controller for a magnetic levitation system
}

\author{
Omar Waleed Abdulwahhab \\ Department of Computer Engineering, University of Baghdad, Iraq
}

\begin{tabular}{l} 
Article Info \\
\hline Article history: \\
Received Feb 12, 2020 \\
Revised Mar 10, 2020 \\
Accepted Mar 25, 2020 \\
\hline
\end{tabular}

\section{Keywords:}

Adaptive state feedback controller

Indirect lyapunov's theorem Magnetic levitation system Unstable nonlinear system

\begin{abstract}
This paper presents designing an adaptive state feedback controller (ASFC) for a magnetic levitation system (MLS), which is an unstable system and has high nonlinearity and represents a challenging control problem. First, a nonadaptive state feedback controller (SFC) is designed by linearization about a selected equilibrium point and designing a SFC by pole-placement method to achieve maximum overshoot of $1.5 \%$ and settling time of $1 \mathrm{~s}(5 \%$ criterion). When the operating point changes, the designed controller can no longer achieve the design specifications, since it is designed based on a linearization about a different operating point. This gives rise to utilizing the adaptive control scheme to parameterize the state feedback controller in terms of the operating point. The results of the simulation show that the operating point has significant effect on the performance of nonadaptive $\mathrm{SFC}$, and this performance may degrade as the operating point deviates from the equilibrium point, while the ASFC achieves the required design specification for any operating point and outperforms the state feedback controller from this point of view.
\end{abstract}

Copyright $(0) 2020$ Institute of Advanced Engineering and Science. All rights reserved.

\section{Corresponding Author:}

Omar Waleed Abdulwahhab, Department of Computer Engineering, University of Baghdad, Iraq.

Email: omar.waleedl@coeng.uobaghdad.edu.iq

\section{INTRODUCTION}

Magnetic levitation technology has recently become an interesting topic of study, since it is a good solution for many motion systems [1,2]. The advantages of a MLS are its abilities to eliminate friction by eliminating the contact between moving and stationary parts [3], decreasing the cost of maintenance, and achieving precise position [4]. The MLSs has become suitable for trains, bearings, vibrating isolation systems, and levitation of wind tunnel $[1,4]$.

By magnetic levitation, a ferromagnetic mass is suspended in the air by an electric magnetic field [5]. The basic control aim is to precisely position the levitating object [6]. To stabilize the MLS, the magnetic field strength must be varied by changing the current of the coil [5, 7]. Since the MLS is unstable and has high nonlinearity, designing a controller for this system with adequate specifications is not a trivial task; thus, the control of this system has received considerable interest [4], and it has become a platform to test different control algorithms $[1,5]$.

Several control approaches were used to stabilize the MLS, such as feedback linearization [8-10], which requires an accurate model of this system; however, obtaining an accurate model represents a problem because of the high nonlinearity of this system and the variation of the gain parameter with the distance between the levitating object and the magnet. Linearization-based methods were also used, where the system is linearized about a certain equilibrium point and a controller is designed to stabilize the system, such as PID controller [1, 2, 5, 6, 7, 11], fractional order PID controller [4, 12-15], LQR [1, 2, 16, 17], lead compensator [1], H_ $\infty$ controller [18, 19], fuzzy logic controller (FLC) [16, 20, 21], and adaptive FLC [22]; however, the performance of such controllers degrade when the deviation between the operating 
point and the equilibrium point (the point that the system was linearized about) increases. To handle this problem, sliding mode controller (SMC) [23-25], adaptive SMC [26], PID-notch filters [27], and linearization-gain scheduling controller PID controller [28], linearization-gain scheduling PI controller [29], and linearization-adaptive PD controller [30] were designed to provide robustness against operating point variation. This paper proposes an ASFC to stabilize the MLS, where the controller parameters become a function of the operating point, and pole placement method is used to design the controller. The rest of this paper is: section 2 presents the mathematical model of the MLS, section 3 presents the design of an ASFC for this system by pole placement, simulation results and discussions are given in section 4, and finally the conclusions that can be drawn from the obtained results are given in section 5 .

\section{MATHEMATICAL MODEL OF THE MLS}

In a MLS, a ferromagnetic ball is levitated by a magnetic field, and the ball position is fed back to control the current of the coil [31]. The position of the ball is

$$
m \ddot{y}=-k \dot{y}+m g+F(y, i)
$$

where $m$ and $y$ are the mass the vertical position of the ball, $\mathrm{k}$ is a viscous friction coefficient, $g$ is the gravity acceleration, $F(y, i)$ is the electromagnet force, and $i$ is the coil current [31]. The inductance which is a function of the ball position is approximately

$$
L(y)=L_{1}+\frac{L_{0}}{1+\frac{y}{a}}
$$

where $L_{1}$ is the electromagnetic coil inductance without the suspended ball, $L_{0}$ is the inductance due to the ball, and $a$ is the air gap when the levitated ball is in equilibrium [32]. The inductance has its highest value $L_{1}+L_{0}$ as the ball touches the magnet and decreases to $L_{1}$ when it is removed away from the coil. If $E(y, i)=\frac{1}{2} L(y) i^{2}$ is the electromagnet stored energy, the force $\mathrm{F}$ is [31]

$$
F(y, i)=\frac{\partial E}{\partial y}=-\frac{L_{0} i^{2}}{2 a\left(1+\frac{y}{a}\right)^{2}}
$$

The magnetic flux linkage is

$$
\phi=L(y) i
$$

and according to Kirchhoff's voltage law, the coil voltage is

$$
v=\dot{\phi}+R i
$$

where $R$ is the circuit resistance. Using $x_{1}=y, x_{2}=\dot{y}$, and $x_{3}=i$ as state variables, $u=v$ as control input, and $y$ as the controlled output, the state matrix equation and the output equation become:

$$
\begin{aligned}
& \dot{\boldsymbol{x}}=\left[\begin{array}{c}
x_{2} \\
g-\frac{k}{m} x_{2}-\frac{L_{0} a x_{3}^{2}}{2 m\left(a+x_{1}\right)^{2}} \\
\frac{1}{L\left(x_{1}\right)}\left(-R x_{3}+\frac{L_{0} a x_{2} x_{3}}{\left(a+x_{1}\right)^{2}}+u\right)
\end{array}\right]=\boldsymbol{f}(\boldsymbol{x}, u) \\
& y=x_{1}
\end{aligned}
$$

The equilibrium point of system (6) can be found by setting $\dot{\boldsymbol{x}}=0$. If this point is designated by $\left(\boldsymbol{x}_{s s}, u_{s s}\right)$ where $\boldsymbol{x}_{s s}=\left[x_{1 s s} x_{2 s s} x_{3 s s}\right]^{T}=\left[r, x_{2 s s}, I_{s s}\right]^{T}$, and $u_{s s}=V_{s s}$, then

$$
\begin{aligned}
& 0=x_{2 \mathrm{ss}}, \\
& 0=g-\frac{k}{m} x_{2 \mathrm{ss}}-\frac{L_{0} a I_{\mathrm{Ss}}}{2 m(a+r)^{2}} \\
& 0=\frac{1}{L(r)}\left(-R I_{\mathrm{ss}}+\frac{L_{0} a x_{2} I_{\mathrm{ss}}}{2 m(a+r)^{2}}+V_{\mathrm{ss}}\right)
\end{aligned}
$$

Solving (8)-(10) for $r, x_{2 s s}$, and $I_{s s}$ in terms of $V_{s s}$ yields 


$$
\left[r, x_{2 \mathrm{ss}}, I_{\mathrm{ss}}\right]^{T}=\left[\frac{1}{R} \sqrt{\frac{L_{0} a}{2 m g}} V_{\mathrm{ss}}-a, 0, \frac{V_{\mathrm{ss}}}{R}\right]^{T}
$$

The linearization of system (6) about the equilibrium point $\left(x_{s s}, u_{s s}\right)$ is

$$
\dot{\boldsymbol{x}}=\boldsymbol{A} \boldsymbol{x}+\boldsymbol{B} u
$$

where $\boldsymbol{A}=\left.\frac{\partial \boldsymbol{f}}{\partial \boldsymbol{x}}\right|_{\left(\boldsymbol{x}_{s,}, u_{s s}\right)}$ and $\boldsymbol{B}=\left.\frac{\partial \boldsymbol{f}}{\partial u}\right|_{\left(\boldsymbol{x}_{s s}, u_{s s}\right)}$. For any equilibrium point $\left(\boldsymbol{x}_{s s}, u_{s s}\right)$, at least one of the three eigenvalues of matrix $\boldsymbol{A}$ has positive real part. Thus, by indirect Lyapunov's Theorem, the system is unstable. The values of the parameters of the MLS are given in Table 1.

Table 1. Parameters of the MLS

\begin{tabular}{lll}
\hline Parameter & Description & Value \\
\hline$m$ & Mass of the ball & $0.1 \mathrm{~kg}$ \\
$k$ & Viscous friction coefficient & $0.001 \mathrm{~N} / \mathrm{m} / \mathrm{s}$ \\
$g$ & Gravity acceleration & $9.81 \mathrm{~m} / \mathrm{s}^{2}$ \\
$a$ & Air gap when the levitated ball is in equilibrium & $0.05 \mathrm{~m}$ \\
$L_{0}$ & Inductance due to levitated ball & $0.01 \mathrm{H}$ \\
$L_{1}$ & Electromagnetic coil inductance without the suspended ball & $0.02 \mathrm{H}$ \\
$R$ & Series resistance of the circuit & $1 \Omega$ \\
\hline
\end{tabular}

\section{CONTROLLER DESIGN}

To demonstrate the enhanced performance of the proposed ASFC, a nonadaptive SFC

$$
u_{f}=-\boldsymbol{K} \boldsymbol{x}=\left[\begin{array}{lll}
k_{1} & k_{2} & k_{3}
\end{array}\right]\left[\begin{array}{l}
x_{1} \\
x_{2} \\
x_{3}
\end{array}\right]
$$

Is designed to stabilize the closed loop system at $r=0.04 \mathrm{~m}$, which corresponds to the equilibrium point $\left(\boldsymbol{x}_{s s}, u_{s s}\right)=\left(\left[\begin{array}{c}0.04 \\ 0 \\ 5.6377\end{array}\right], 5.6377\right)$. For this equilibrium point, the linear system (12) becomes

$$
\dot{\boldsymbol{x}}=\left[\begin{array}{ccc}
0 & 1 & 0 \\
218 & -0.1 & -3.4801 \\
0 & 13.6178 & -39.13304
\end{array}\right] \boldsymbol{x}+\left[\begin{array}{c}
0 \\
0 \\
39.13304
\end{array}\right] u
$$

The gain matrix $\boldsymbol{K}$ is designed to locate the closed loop poles at positions so that the percentage overshoot is $1.5 \%$ and the settling time is $0.5 \mathrm{~s}$ ( $5 \%$ criterion).

$$
M_{P}=e^{\frac{-\xi \pi}{\sqrt{1-\xi^{2}}}} \Rightarrow \frac{1.5}{100}=e^{\frac{-\xi \pi}{\sqrt{1-\xi^{2}}}} \Rightarrow \xi=0.8 \text { and } T_{\mathrm{s}}=\frac{3}{\xi \omega_{n}} \Rightarrow 0.5=\frac{3}{0.8 \omega_{n}} \Rightarrow \omega_{n}=7.5 \frac{\mathrm{rad}}{\mathrm{s}}
$$

Thus, the two complex conjugate poles are $-6 \pm \mathrm{j} 4.5$. To make the poles $s_{1,2}$ dominant, the third pole is selected such that $\left|\operatorname{Re}\left(s_{3}\right)\right| \geq 5\left|\operatorname{Re}\left(s_{1,2}\right)\right|$; let $s_{3}=-30$. Using Ackerman's formula, the gain matrix is

$$
\begin{aligned}
\boldsymbol{K} & =\left[\begin{array}{lll}
0 & 0 & 1
\end{array}\right]\left[\boldsymbol{B} \boldsymbol{A B} \boldsymbol{A}^{2} \boldsymbol{B}\right]^{-1}((\boldsymbol{A}-(-6+j 4.5) \boldsymbol{I})(\boldsymbol{A}-(-6-j 4.5) \boldsymbol{I})(\boldsymbol{A}-(-30) \boldsymbol{I})) \\
& =\left[\begin{array}{lll}
-79.6114-4.30640 .0731
\end{array}\right]
\end{aligned}
$$

and the control law becomes

$$
u=u_{f}+u_{s s}=-79.6114 x_{1}-4.3064 x_{2}-0.0731 x_{3}+5.6377
$$

A block diagram of the MLS with SFC is shown in Figure 1. A drawback of this controller is that it assures the stabilization of the system and it achieves the required design specifications only in a certain neighborhood of the linearization-based point, i.e., the equilibrium point that corresponds to $r=0.04 \mathrm{~m}$. To stabilize the system at another position, the controller may fail to stabilize the system, or at least it will not achieve the required design specifications. 
To overcome this problem, an adaptive state feedback controller is designed. This can be achieved by parameterizing the linear system (14) in terms of its equilibrium point, i.e., the quantities $\boldsymbol{x}_{s s}=\left[\begin{array}{lll}x_{1 s s} & x_{2 s s} & x_{3 s s}\end{array}\right]^{T}$ and $u_{s s}=V_{s S}$ are not given constant values; rather, they are considered as parameters, and system (14) can be rewritten as

$$
\begin{aligned}
& \dot{\boldsymbol{x}}=\boldsymbol{A}\left(\boldsymbol{x}_{\mathrm{ss}}, u_{\mathrm{ss}}\right)+\boldsymbol{B}\left(\boldsymbol{x}_{\mathrm{ss}}, u_{\mathrm{ss}}\right) u \\
& \boldsymbol{x}_{\mathrm{ss}}=\left[\begin{array}{c}
1 \\
0 \\
\sqrt{\frac{L_{0} a}{2 m g}}
\end{array}\right] r+\left[\begin{array}{c}
0 \\
0 \\
\sqrt{\frac{L_{0} a}{2 m g}} a
\end{array}\right]=\boldsymbol{g}_{\boldsymbol{x}}(r) \\
& u_{\mathrm{ss}}=R \sqrt{\frac{L_{0} a}{2 m g}}(a+r)=g_{u}(r)
\end{aligned}
$$

and system (17) can be rewritten as

$$
\dot{\boldsymbol{x}}=\boldsymbol{A}(r)+\boldsymbol{B}(r) u
$$

and the ASFC is

$$
u=-\boldsymbol{K}(r) \boldsymbol{x}=\left[\begin{array}{lll}
k_{1}(r) & k_{2}(r) \quad k_{3}(r)
\end{array}\right]\left[\begin{array}{l}
x_{1} \\
x_{2} \\
x_{3}
\end{array}\right]
$$

where $K(r)$ is given by

$$
\begin{aligned}
& \boldsymbol{K}(r)=\left[\begin{array}{lll}
0 & 0 & 1
\end{array}\right]\left[\boldsymbol{B}(r) \boldsymbol{A}(r) \boldsymbol{B}(r)(\boldsymbol{A}(r))^{2} \boldsymbol{B}(r)\right]^{-1} \\
& ((\boldsymbol{A}-(-6+j 4.5) \boldsymbol{I})(\boldsymbol{A}-(-6-j 4.5) \boldsymbol{I})(\boldsymbol{A}-(-30) \boldsymbol{I}))
\end{aligned}
$$

The control law (21) is a family of controllers, i.e., an adaptive state feedback controller, whose parameters $k_{1}, k_{2}$, and $k_{3}$ are changed (designed) according to the value of the reference input $r$. A block diagram of the MLS with ASFC is shown in Figure 2.

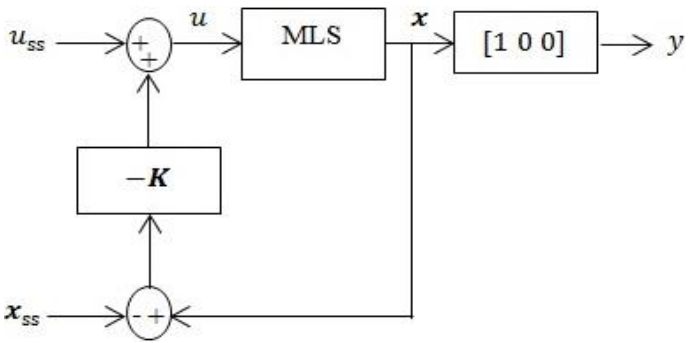

Figure 1. Block diagram of the MLS with SFC

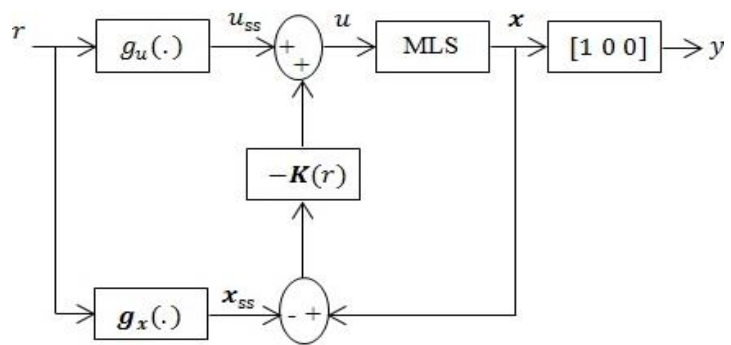

Figure 2. Block diagram of the MLS with ASFC

\section{SIMULATION RESULTS AND DISCUSSION}

A simulation of the closed loop MLS was carried out using script MATLAB program. Four cases were considered, regarding the operating range of the system. The first case is when the system operates in a range that lies relatively close to the equilibrium point that corresponds to $r=0.04 \mathrm{~m}$; this range was achieved by taking an initial position $y_{0}=0.02 \mathrm{~m}$ and a desired position $r=0.06 \mathrm{~m}$. The second case is when the system operates in a range that deviates from the equilibrium point by a relatively moderate distance; this range was achieved by taking an initial position $y_{0}=0.06 \mathrm{~m}$ and a desired position $r=0.10 \mathrm{~m}$. The third case is when the system operates in a range that deviates from the equilibrium point by a relatively large distance; this range was achieved by taking an initial position $y_{0}=0.10 \mathrm{~m}$ and a desired position $r=0.14 \mathrm{~m}$. The fourth case is when the system operates in a wide range; this range was achieved by taking an initial position $y_{0}=0.01 \mathrm{~m}$ and a desired position $r=0.10 \mathrm{~m}$. Figure 3 shows the ranges of the operating points of the four cases relative to the linearization-based point, and Table 2 shows the performance of the system with the SFC and with the ASFC, for all cases. 


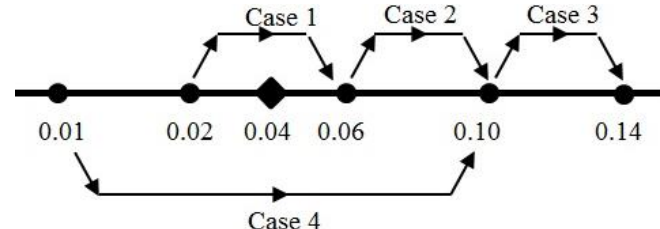

Linearization-based point

Initial/desired point

Figure 3. Ranges of operating points of the four cases
Table 2. Performance of the system

\begin{tabular}{ccccc}
\hline & & $\begin{array}{c}\text { Rise time } \\
(\mathrm{s})\end{array}$ & $\begin{array}{c}\text { Settling } \\
\text { time (s) }\end{array}$ & $\begin{array}{c}\text { Percentage } \\
\text { overshoot }\end{array}$ \\
\hline Case 1 & SFC & 0.26 & 0.94 & $3.30 \%$ \\
& ASFC & 0.29 & 0.58 & $0.73 \%$ \\
Case 2 & SFC & 0.28 & 1.10 & $4.92 \%$ \\
& ASFC & 0.31 & 0.54 & $0.47 \%$ \\
Case 3 & SFC & 0.30 & 1.21 & $5.08 \%$ \\
& ASFC & 0.32 & 0.51 & $0.35 \%$ \\
Case 4 & SFC & Unstable & & \\
& ASFC & 0.34 & 0.69 & $0.53 \%$ \\
\hline
\end{tabular}

The results given in Table 2 shows that as the operating point deviates from the linearization-based point, the performance of the SFC degraded (the rise time, the settling time, and the percentage overshoot are increased), and it became unstable in the fourth case. However, the ASFC showed better performance and robustness, since the controller gain matrix was adapted with every new reference input to maintain the same required design specifications of the system. The responses of both controllers for the four cases are shown in Figures 4-11.
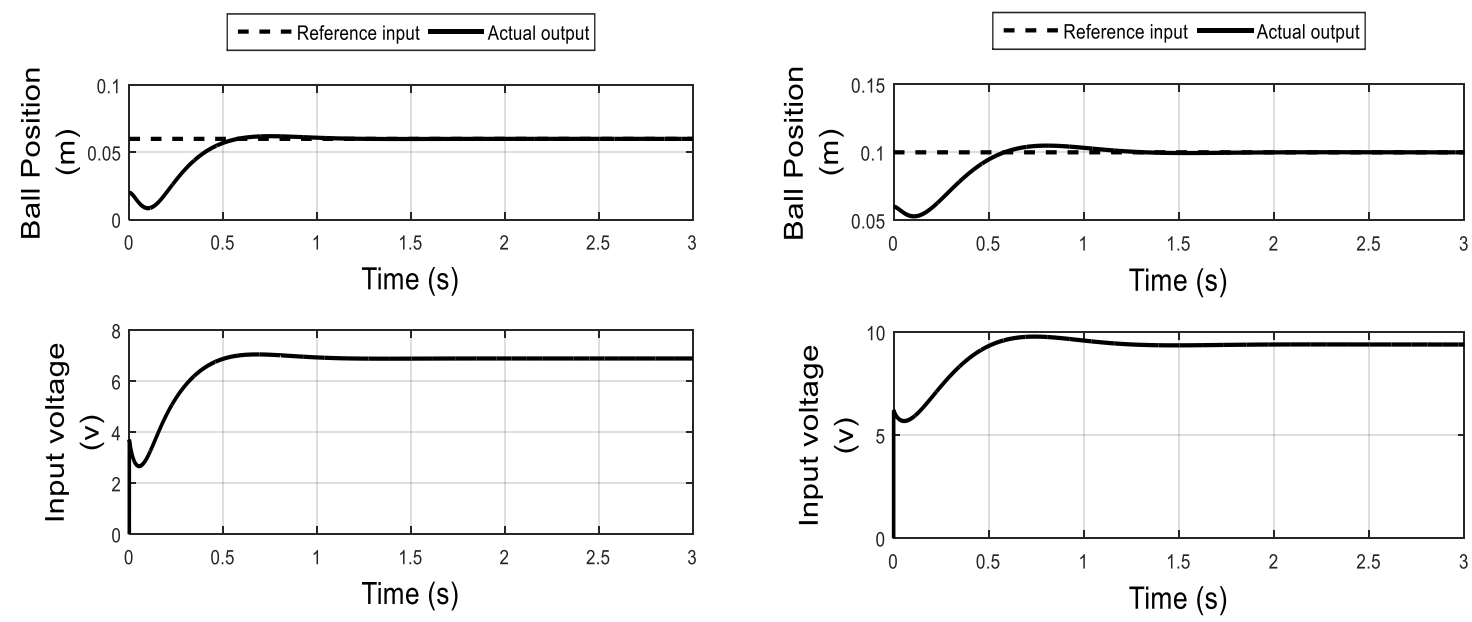

Figure 4. Step response of MLS with SFC: case 1

Figure 5. Step response of MLS with SFC: case 2
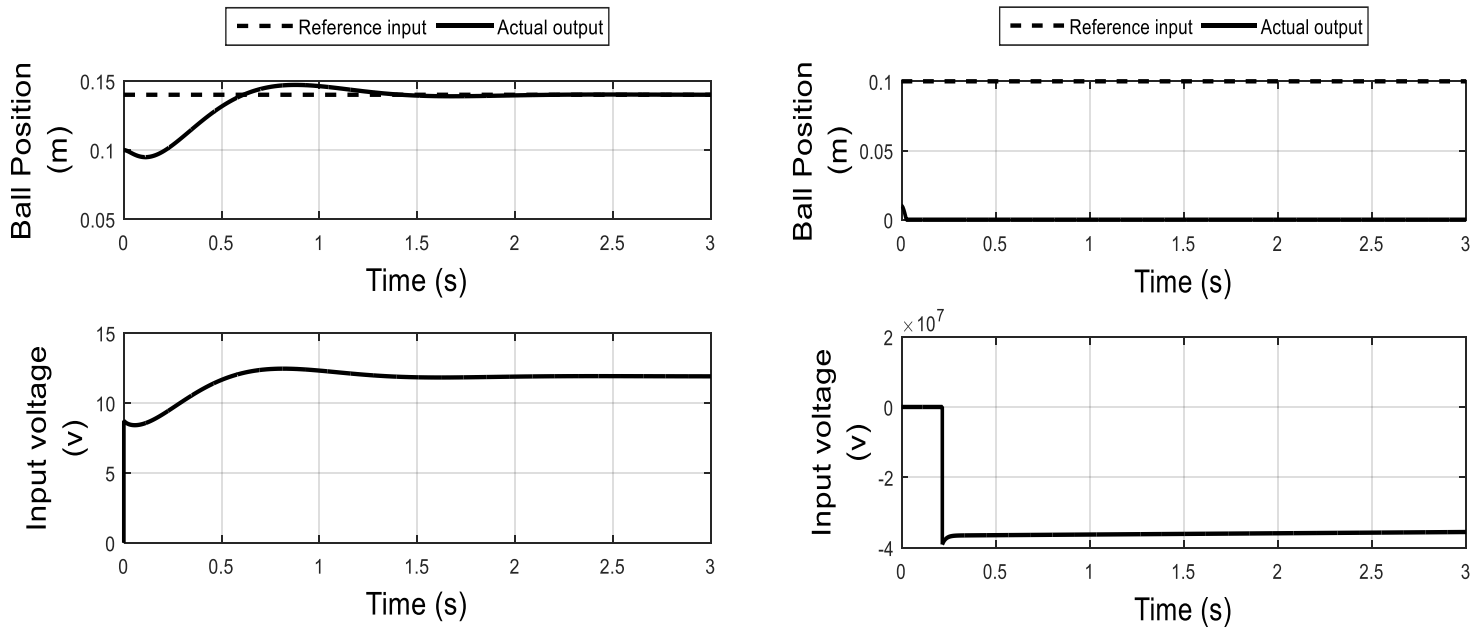

Figure 6. Step response of MLS with SFC: case 3

Figure 7. Step response of MLS with SFC: case 4, 

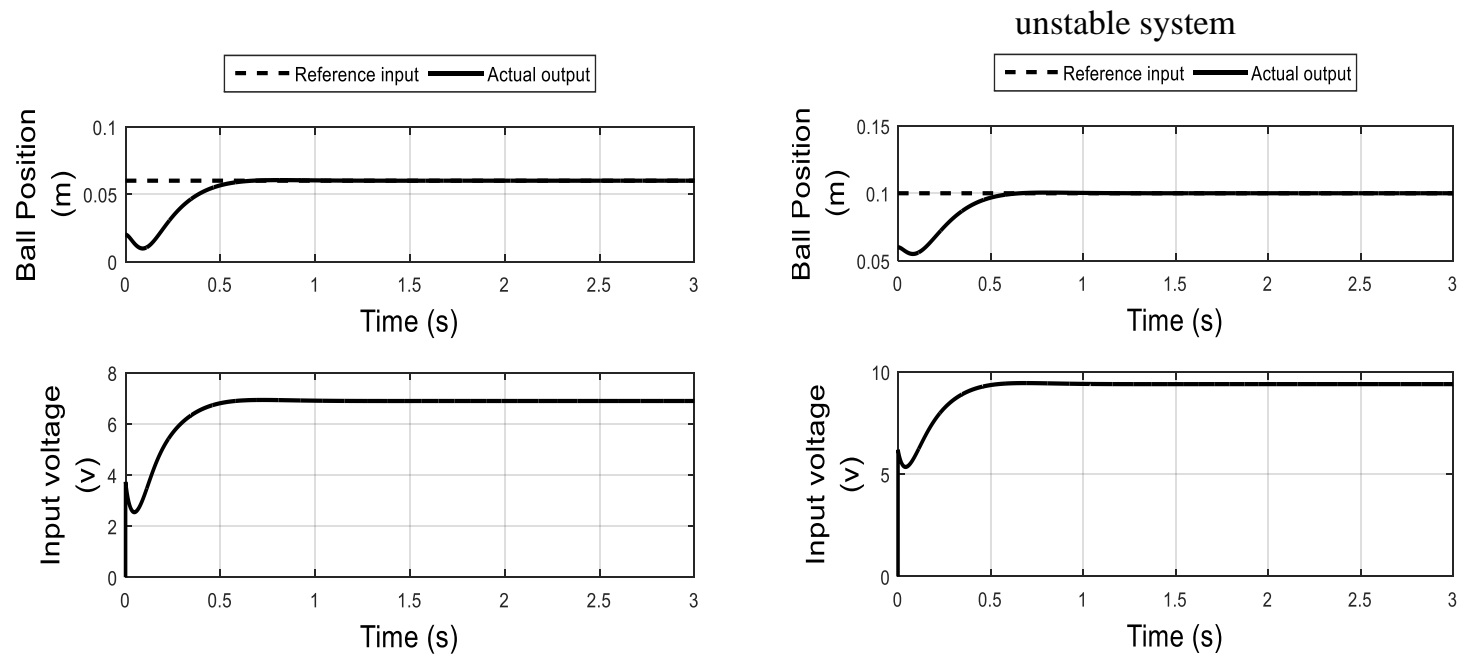

Figure 8. Step response of MLS with ASFC: case 1

Figure 9. Step response of MLS with ASFC: case 2
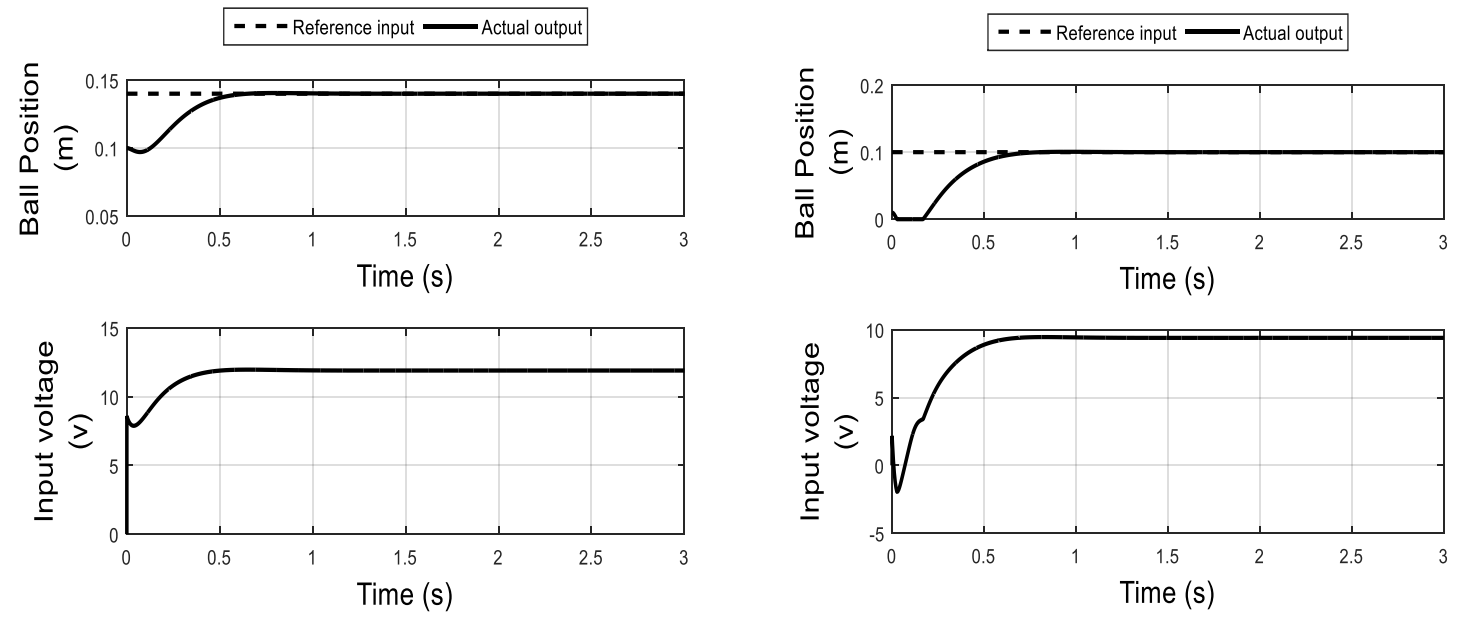

Figure 10. Step response of MLS with ASFC: case 3 Figure 11. Step response of MLS with ASFC: case 4

\section{CONCLUSION}

In this paper, the design of an ASFC for a MLS has been proposed. The SFC was design by first linearizing the MLS about a selected equilibrium point, then the closed loop poles are positioned at locations so as to achieve certain design specification. However, when the reference input changed, the nonadaptive state feedback controller could no longer satisfy the closed loop design specifications and its performance degraded, or even it fails to stabilize the MLS, while the ASFC satisfied the closed loop design specifications for all reference inputs. Several conclusions can be drawn from the obtained results. First, the linearization design method has a limitation when applied to highly nonlinear system, such as the MLS. Second, the ASFC is a suitable solution to stabilize highly nonlinear system, and it outperforms the nonadaptive state feedback controller.

\section{REFERENCES}

[1] M. H. A. Yaseen and H. J. Abd, "Modeling and control for a magnetic levitation system based on SIMLAB platform in real time," Results in Physics, vol. 8, pp. 153-159, 2018.

[2] M. H. A. Yaseen and H. J. Abd, "A new planar electromagnetic levitation system improvement method based on SIMLAB platform in real time operation," Progress In Electromagnetic Research, vol. 62, pp. 211-221, 2017.

[3] W. Wiboonjaroen and S. Sujitjorn, "Stabilization of a magnetic levitation control system via state-PI feedback," International Journal of Mathematical Models and Methods in Applied Science, vol. 7, no. 7, 2013.

[4] S. Folea, et al., "Theoretical analysis and experimental validation of a simplified fractional order controller for a magnetic levitation system," IEEE Transactions on Control Systems Technology, vol. 24, no. 2, pp. 756-763, 2016. 
[5] A. Duka, et al., "IMC based PID control of a magnetic levitation system," Procedia Technology, vol. 22, 2016.

[6] P. Balko and D. Rosinová, "Modeling of magnetic levitation system," in 21st International Conference on Process Control (PC), Strbske Pleso, Slovakia, 2017.

[7] P. Rajalakshmy, et al., "Development of magnetic levitation system," International Journal of Innovative Technology and Exploring Engineering, vol. 8, no. 4, 2019.

[8] R. Morales, et al., "Adaptive control based on fast online algebraic identification and GPI control for magnetic levitation systems with time-varying input gain," International Journal of Control, vol. 87, no. 8, pp. 1604-21, 2014.

[9] L. Jin-hui, et al., "A practical robust nonlinear controller for maglev levitation system," Journal of Central South University, vol. 20, no. 11, 2013.

[10] I. Ahmad and M. A. Javaid, "Nonlinear model and controller design for magnetic levitation system," in ISPRA'10 Proceedings of the 9th WSEAS international conference on Signal processing, robotics and automation 2010, UK.

[11] S. Yadav, et al., "Performance enhancement of magnetic levitation system using teaching learning based optimization," Alexandria Engineering Journal, vol. 57, no. 4, pp. 2427-33, 2018.

[12] A. Tepljakov, et al., "Design of retuning fractional PID controllers for a closed-loop magnetic levitation control system," in 2014 13th International Conference on Control Automation Robotics and Vision - 2014 ICARCV, Singapore, 2014.

[13] J. Zong and L. Li, "Tuning Fractional-Order $\mathrm{PI}^{\lambda} \mathrm{D}^{\mu}$ Controllers for a solid-Core Magnetic Bearing System,” IEEE Transactions on Control Systems Technology, vol. 23, no. 4, pp. 1648-1656, 2015.

[14] H. Gole, et al., "Investigation of fractional control performance for magnetic levitation experimental set-up," in 2012 International Conference on Emerging Trends in Science, Engineering and Technology (INCOSET), Tiruchirappalli, India, 2012.

[15] N. Sadati, "Optimum design of fractional order PID for MIMO and SISO Systems using particle swarm optimization techniques," in Proceeding of International Conference on Mechatronics, Kumamoto, Japan, 2007.

[16] T. Salim and V. M. Karsli, "Control of single axis magnetic levitation system using fuzzy logic control," International Journal of Advanced Computer Science and Applications, vol. 4, no. 11, pp. 83-88, 2013.

[17] A. Suebsomran, "Optimal control of electromagnetic suspension EMS system," The open Automation and Control Systems Journal, vol. 6, no. 1, pp. 1-8, 2014.

[18] S. K. Choudhary, "Robust feedback control analysis of magnetic levitation system," WSEAS Transactions on Systems, vol. 13, pp. 285-291, 2014.

[19] Z. Yanhong, et al., "Robustness of improving active maglev motorized spindle equilibrium position," TELKOMNIKA Indonesian Journal of Electrical Engineering and Computer Science, vol. 11, no. 9, pp. 5161-68, 2013.

[20] K. Ishaque, et al., "Modeling and control of magnetic system via fuzzy logic controller," in 2011 Fourth International Conference on Modeling, Simulation and Applied Optimization, Kuala Lumpur, Malaysia 2011.

[21] D. S. Shu'aibu, et al., "Efficient fuzzy logic controller for magnetic levitation systems," Nigerian Journal of Technological Development, vol. 13, no. 2, pp. 50-57, 2016.

[22] J. J. Hernández-Casañas, et al., "Characterization and adaptive fuzzy model reference control for a magnetic levitation system," Alexandria Engineering Journal, vol. 55, no. 3, pp. 2597-2607, 2016.

[23] E. T. Moghaddam and J. Ganji, "Sliding mode control of magnetic levitation systems using hybrid extended Kalman filter," Energy Science and Technology, vol. 2, no. 2, pp. 35-42, 2011.

[24] B. Krishna and D. Chandran, "Sliding mode control for magnetic levitation system: an eigen structure assignment approach," in 9th Asian Thermal Spray Conference, 2018.

[25] S. A. Al-Samarraie, "Variable Structure Control Design for a Magnetic Levitation System," Journal of Engineering, vol. 24, no. 12, pp. 84-103, 2018.

[26] S. A. Al-Samarraie, et al., "Adaptive sliding mode control for magnetic levitation system," Al-Nahrain Journal for Engineering Sciences (NJES), vol. 21, no. 2, pp. 266-74, 2018.

[27] A. Noshadi, et al., "A Genetic algorithm-based system identification of active magnetic bearing system: a frequency-domain approach," in 11th IEEE International Conference on Control \& Automation (ICCA), Taichung, Taiwan 2014,.

[28] C.-A. Bojan-Dragos, et al., "Proportional-integral-derivative gain-scheduling control of a magnetic levitation system," International Journal of Computers Communications \& Control, vol. 12, no. 5, pp. 599-611, 2017.

[29] C.-A Bojan-Dragos, et al., "Gain-scheduling control solutions for magnetic levitation systems," Acta Polytechnica Hungarica, vol. 15, no. 5, pp. 89-108, 2018.

[30] Z. Zhang and X. Li, "Real-time adaptive control of a magnetic levitation system with a large range of load disturbance," Sensors, vol. 18, no. 5, 2018.

[31] H. K Khalil, Nonlinear Systems, 3rd ed, USA: Prentice Hall: 2002.

[32] H. J. Marquez, Nonlinear Control systems. New Jersey: John Wiley \& Sons, 2003. 